BLS 35, No 1 2009. DOI: http://dx.doi.org/10.3765/bls.v35i1.3595

(published by the Berkeley Linguistics Society and the Linguistic Society of America)

\title{
Promiscuous Modification and Cross-Categorial Scale Structure
}

\author{
M. RYAN BOCHNAK \\ University of Chicago
}

\section{Introduction}

I use the term "promiscuous modification" to refer to the two-fold properties of certain proportional modifiers in English and other languages: first, they are able to modify various syntactic and semantic categories; second, they appear to be able to modify at a distance. That is, while they can adjoin to one constituent syntactically they appear to modify another semantically. The focus of this paper is the modifier half in English, which indeed fits the description of promiscuous modification. First, it can appear with a wide range of syntactic categories, such as adjectives in (1), partitives as in (2), and verb phrases as in (3):

(1) The cake is half baked.

(2) Half of the books are on the table.

(3) The girls half washed the dishes.

Second, half has the ability to modify at a distance. For instance, the sentence in (1) has two distinct readings. On one reading it describes a situation where the cake is half-way through the process of baking, in which case we can say that half is modifying the adjective baked, in particular the scale associated with the adjective baked (see Kennedy and McNally 2005). On the second reading, (1) describes a situation where half of the cake is fully baked and the other half is not baked at all. For example, it might be the case that a cake is too large to fit into an oven and must be divided into two halves that are baked separately. In this case half appears to be modifying the cake at a distance, in that it names the proportion of the cake that is baked. However, there are constraints on these long-distance effects. Notice that in (3) a long-distance reading is possible where half appears to be modifying the object the dishes at a distance, although there is no reading where half modifies the subject the girls at a distance.

The focus of the present paper will be to argue for an account of these longdistance effects based on argument structure and event structure of the verb phrase. I will propose a scalar-based semantics for half that allows for and explains the cross-categorial nature of half. In particular I claim that the promiscu- 


\section{Ryan Bochnak}

ous behavior of half is the result of its scalar meaning, and that the successful application of this modifier simply relies on its input being associated with an appropriate scale over which half can operate. In section $1 \mathrm{I}$ argue that half is a modifier at the VP level (contra Piñón 2005) and that the long-distance effects can be explained in terms of argument selection. Section 2 is devoted to showing that half is a modifier of telic event descriptions. In section 3 the semantics of the VP use of half is fleshed out in more detail. Section 4 concludes and offers proposals for future extensions of this work.

\section{Verb Phrase Modification and Argument Structure}

One possible explanation of the long-distance effects associated with half is to say that this is an instance of floating quantification (see, e.g., Sportiche 1988). The idea would be that in (1) half and the cake form a constituent at some level of underlying representation and the cake undergoes leftward movement, leaving the quantifier behind. This line of argumentation has been used to account for the synonymy of (4a) and (4b), where all as a floating quantifier forms a constituent with the girls at an underlying level:

a. The girls all washed the dishes.

b. All the girls washed the dishes.

However, such an account cannot be the right analysis for half. First, replacing all with half in (4) leads to sentences that are no longer synonymous. Indeed, as has already been observed in (3), half cannot modify the girls at a distance in this environment, and furthermore half is able to modify the dishes at a distance, but a corresponding reading is unavailable for all in (4). Second, while all is able to float through auxiliaries with ease, this is not the case for half as shown by the contrast between (5) and (6):

(5) The dishes $\{$ all $\}$ must $\{$ all $\}$ have $\{$ all $\}$ been $\{$ all $\}$ washed.

(6) The dishes $\{*$ half $\}$ must $\{*$ half $\}$ have $\{$ ?half $\}$ been $\{$ half $\}$ washed.

Based on this evidence, half should not be treated as a floating quantifier on par with all.

Instead, I propose that the facts can best be dealt with by analyzing half as a VP modifier. In particular, the long-distance effects associated with half are sensitive to the distinction between a verb's internal and external arguments. Kratzer (2003) provides extensive evidence exposing asymmetries between a verb's external and internal arguments (also see Tenny 1994). Without going into too much detail, I will briefly review a couple of Kratzer's arguments here. The first piece of evidence comes from deverbal nominalizations that require a verb's internal argument to be realized. Compare (7a) with (7b): 


\section{Promiscuous Modification and Scale Structure}

a. *The constant assignment is to be avoided.

b. The constant assignment of unsolvable problems is to be avoided.

The ungrammaticality of (7a) is due to the fact that the internal argument of the verb assign must be realized within the nominalization. However, since the verb's external argument is not (overtly) required in its nominalization, this means that it does not have the same effects on verb phrase meaning as the internal argument does.

The second asymmetry between internal and external arguments comes from constructions that allow or disallow self-action readings. Comparing the adjectival passive in (8a) and the verbal passive in (8b), we notice that while neither explicitly realizes the verb's external argument, only the adjectival passive is compatible with a reading where the climbers secured themselves; this reading is unavailable with the verbal passive:

a. The climbers are secured with a rope.

b. The climbers are being secured with a rope.

Kratzer argues that in verbal passives, the external argument, while not overtly expressed, is still present as a silent pronoun, which accounts for why (8b) is incompatible with a self-action reading. In contrast, the adjectival passive in (8a) allows a self-action reading, which means that the external argument of the verb can be missing altogether. This contrast is taken as further evidence that the external argument does not serve the same function in verb phrase meaning as the internal argument, and therefore these two types of arguments are not associated with the verb in the same way. In fact, this evidence is taken to suggest that the external argument is not even a real argument of the verb.

These and other arguments show not only that the internal and external arguments of a verb need to be treated differently, but that the external argument is not even part of the verb phrase. Kratzer argues that this treatment of the external argument is necessary both at the level of logical representation and in the syntax. The proposal, then, is that instead of being introduced with the verb phrase, the external argument enters the syntax inside a voice (little $v$ ) projection. This analysis explains why adjectival passives and some nominalizations there is no external argument present: these are derived from VP only, which contains only the verb and its internal argument but crucially not the external argument.

Returning to the discussion on half, recall the contrast between (1) and (3), repeated here as (9) and (10):

(9) The cake is half baked.

(10) The girls half washed the dishes.

Earlier it was noted that sentences like (9) allow a reading where half semantically modifies the subject, but sentences like (10) do not allow this option. This 


\section{Ryan Bochnak}

difference in behavior can now be explained if we take half to be a modifier at the VP level. If the adjectival passive in (9) is derived from a VP structure, as Kratzer argues, then the cake is actually the internal argument of the verb bake. In contrast, the external argument the girls in (10) is introduced outside the VP. In that case, if half is a VP modifier, then this explains its inability to modify the girls: this argument is introduced outside the VP, and is therefore unavailable for modification by half. This prediction is indeed borne out in nominalizations and adjectival passives with half:

(11) the half washing of the dishes (by the girls)

(12) The dishes are half washed.

In (11) the external argument the girls is optional and is introduced with a by phrase and there is no reading available where half semantically modifies this constituent. In (12) there is no external argument present, but there is a reading where half appears to modify the dishes, which is the internal argument of wash.

Analyzing half as a modifier at the VP level easily accounts for the asymmetries observed between (9) and (10) with respect to long-distance effects with half. (9) is derived from a VP structure where the cake is the internal argument of the verb bake, which is under the scope of half. In (10) the dishes is the internal argument of wash and thus under the scope of half, while the girls is introduced into the syntax and semantics outside of VP and outside the scope of half. Thus, the first piece of the puzzle with respect to long-distance modification is solved. A verb's internal argument may be targeted for modification by half, but its external argument may not. The semantics of half that allow it to access the internal argument for modification are discussed below in section 3 .

\section{Modifying Event Descriptions}

I have just shown that long-distance effects associated with half (or lack thereof) can be explained by analyzing half as a modifier of the verb phrase. However, half is not able to modify just any VP. Compare the sentences in (13), which seem odd (on the relevant reading), with those in (14): ${ }^{1}$
a. ?? Alana half ate pancakes.
b. ?? Keelin half swam.
c. ?? Taylor half pushed the cart.
a. Alana half ate a stack of pancakes.
b. Keelin half swam around the lake.
c. Taylor half pushed the cart to the store.

\footnotetext{
${ }^{1}$ The sentences in (13) do have interpretations where half is used to make an evaluative statement about the quality of the event named by the verb, and result in atelic interpretations only. I set aside this reading for now, but see Bochnak (2009) for discussion.
} 


\section{Promiscuous Modification and Scale Structure}

While the sentences in (14) denote telic eventualities, those in (13) have atelic interpretations. Telic events are those that make reference to a particular endpoint, whereas atelic eventualities are those that do not. In particular, telicity has been construed as being related to the boundedness or measuring out of an event (Krifka 1992, 1998). An event may be interpreted as telic if it references a bounded, or "quantized" incremental theme, as argued by Dowty (1991), Krifka (1992), and others. An incremental theme is one whose parts are incrementally affected through the duration of the event. Classic examples of bounded incremental themes are an apple in John ate an apple and a circle in John drew a circle. In the case of an apple for example, it is typically the case that over the course of an event of eating an apple, pieces of the apple are consumed in succession until the entire apple is consumed, or at least the relevant parts. That is, there is a mapping from sub-events to parts of the incremental theme, and also a mapping between the parts of the incremental theme and sub-events. This intuition is captured by Krifka's object-event homomorphism. Thus, while in (14a) $a$ stack of pancakes corresponds to a bounded incremental theme that gives rise to a telic interpretation, pancakes in (13a) is an unbounded quantity, thereby resulting in an atelic interpretation. In (13c) the theme argument the cart does not give rise to a telic interpretation because it is not an incremental theme - the entire cart is affected throughout the duration of the event. In addition, a telic event may also be interpreted as bounded by naming a bounded path, as argued by Jackendoff (1996). Thus (14b-c) have telic interpretations thanks to the bounded path arguments, around the lake and to the store, respectively, while (13b-c) can only have atelic interpretations.

Sentences with incremental themes or bounded paths where half modifies the VP do indeed denote telic events, as evidenced by their acceptability with in adverbials and their oddity with for adverbials:

(15) Alana half ate a stack of pancakes in 10 minutes / ?? for 10 minutes.

(16) Keelin half swam around the lake in 10 minutes / ?? for 10 minutes.

(17) Taylor half pushed the cart to the store in 10 minutes / ?? for 10 minutes.

Thus the application of half as a VP modifier results in telic interpretations when there is a bounded incremental theme or bounded path argument that measures out the event. The result is a reading where half identifies the proportion of the event that is completed. When applied to atelic events half only has an evaluative reading, and not one associated with measuring out events. In the next section I show how bounded themes and path arguments relate to telicity with half.

\section{A Semantics for VP half}

\subsection{Telicity and Maximalization}

Taking the notion of telicity via boundedness one step further, Filip (2008) argues that telicity is the result of a maximalization operation over events. Filip's proposal is that telicity as a property of the VP relies on the application of a maxi- 


\section{Ryan Bochnak}

malization operator over events $\mathrm{MAX}_{\mathrm{E}}$ that maps sets of partially ordered events onto sets of maximal events. This covert operator requires an incremental theme to provide an ordering criterion for events; that is, this operator depends on the object-event homomorphism described above. Filip's notion of incremental theme is taken in a wide sense, including not only theme arguments, but also bounded paths as well as implicit bounded "themes" that are available from the context of utterance or world knowledge. The ordering criterion based on the bounded incremental theme induces a closed scale of objects along which events are ordered, thanks to the object-event homomorphism. The $\mathrm{MAX}_{\mathrm{E}}$ operator selects the endpoint of the closed scale, resulting in a telic interpretation.

The connections between telicity, boundedness and the $\mathrm{MAX}_{\mathrm{E}}$ operator should by now be apparent. Without a bounded theme argument, the scale of objects would have no upper endpoint for $\mathrm{MAX}_{\mathrm{E}}$ to pick out, resulting in an atelic interpretation. This is why unbounded themes such as bare mass or plural nouns result in atelic interpretations - the corresponding scale of objects is an open scale without a maximal value and so does not license the application of $\mathrm{MAX}_{\mathrm{E}}$. Scale closure is also crucial for the successful application of half. As has already been shown, a telic interpretation of a verb phrase modified by half relies on the presence of a bounded incremental theme or path argument. Given that the restrictions on half mirror those of $\mathrm{MAX}_{\mathrm{E}}$ with respect to bounded themes and telicity, I propose that half is in fact an overt counterpart to Filip's $\mathrm{MAX}_{\mathrm{E}}$ operator that relies on the presence of a closed scale of objects induced by a bounded incremental theme. Whereas $\mathrm{MAX}_{\mathrm{E}}$ selects the endpoint on the scale, half selects the midpoint and results in a telic interpretation relative to that midpoint on the scale.

Let us consider a couple of examples to see exactly how this works:

(18) a. The girls washed the dishes.

b. The girls half washes the dishes.

(19) a. The cake is baked.

b. The cake is half baked.

In (18a) the $\mathrm{MAX}_{\mathrm{E}}$ operator selects the endpoint of the scale of objects associated with the bounded incremental theme the dishes and the result is a telic interpretation with respect to the amount of dishes in the denotation of the dishes. Likewise in (18b) half selects the midpoint on the scale of objects and results in a telic interpretation with respect to half of the amount of dishes in the denotation of the dishes. Similarly (19a), being derived from a VP (see section 1), is interpreted as maximal with respect to the quantity of cake, while (19b) is maximal with respect to half of that quantity. Thus, the application of half results in a telic interpretation by maintaining an upper bound against which the event is measured, and that upper bound is the midpoint on the closed scale of objects. 


\section{Promiscuous Modification and Scale Structure}

\subsection{Scales and half}

The idea that half operates over a closed scale is also proposed by Piñón (2005), and is reminiscent of the scalar treatment of half as a modifier of gradable adjectives by Kennedy and McNally (2005). Kennedy and McNally argue that different adjectives are associated with different scale types (open, closed, partially closed) and that half can only modify those adjectives that are associated with fully closed scales. This analysis straightforwardly derives why half is acceptable with adjectives like full, open or baked, which are all associated with fully closed scales, but infelicitous with adjectives like tall, old or expensive, which are associated with open scales:
a. The glass is half full.
b. The door is half open.
c. The cake is half baked.
a. ?? The building is half tall.
b. ?? The man is half old.
c. ?? This car is half expensive.

The fact that half can only apply to adjectives with fully closed scales provides us with some insight into the cross-categorial nature of this modifier. Given its distribution with both adjectives with fully closed scales on one hand and VPs associated with fully closed scales of objects on the other hand, we can say that half is able to modify both adjectives and VPs so long as they are associated with the appropriate type of scale.

We are now in a position to posit a semantics for the VP use of half. Let $F$ be a set of event descriptions associated with a fully closed scale of objects $S_{F}$, and let meas $[\alpha, \beta]$ be a function that returns a measurement of a closed interval $I \subseteq S_{F}$ with endpoints $\alpha$ and $\beta$. The meaning of half is given as follows: ${ }^{2}$

$$
[[\text { half }]]=\lambda F \lambda e . \exists p\left[\operatorname{meas}\left[\min \left(S_{F}\right), \mathrm{p}\right] \geq \operatorname{meas}\left[\mathrm{p}, \max \left(S_{F}\right)\right] \wedge F(e) \text { w.r.t. } p\right]
$$

In prose, half takes a set of event descriptions $F$ as its input and returns another set of event descriptions that are maximal with respect to the midpoint $p$ on the scale of objects induced by the theme in $F$. This analysis explains how we are able to get the long-distance readings with half: the scale of objects is induced by the incremental theme argument but is available for modification at the VP level because it provides the basis for marking telicity, which is a property of the VP. Therefore, both syntactically and semantically, half modifies the VP, but we are able to get readings where half appears to be semantically modifying the theme at

${ }^{2}$ In (22) I give the literal meaning of half as at least half, following the 'classic' neo-Gricean view of the meaning of scalar and number terms (see, e.g., Levinson 2000), but nothing in my analysis really hinges on this assumption. See Ionin and Matushansky (2006) and especially Geurts (2006) for arguments in favor of giving number terms a meaning of exactly $n$. I leave open the question of whether half should be treated as a number term or a scalar term. 


\section{Ryan Bochnak}

a distance because the theme is responsible for inducing the scale of objects over which half operates. This also explains why certain readings are unavailable. For example, in sentences like (18b), half is not able to semantically modify the DP subject the girls because it is the external argument, which is introduced outside the VP and does not induce a scale of objects at the VP level over which half can operate. In addition, when there is no internal argument present, there is no longer a telic reading associated with half due to the lack of a scale of objects from within the VP, and so we only get the evaluative reading in these cases.

To see the meaning of half in action, consider the following derivation for half wash the dishes (ignoring tense):

a. $[[$ wash the dishes $]]=\lambda$ e.wash $($ the.dishes $)(e)$

b. [[ half $]]$ ([[ wash the dishes $]])$

$$
\begin{gathered}
=\lambda F \lambda e . \exists p\left[\operatorname{meas}\left[\min \left(S_{F}\right), \mathrm{p}\right] \geq \operatorname{meas}\left[\mathrm{p}, \max \left(S_{F}\right)\right] \wedge F(e) \text { w.r.t. } p\right] \\
\quad\left(\lambda e^{\prime} \cdot \mathbf{w a s h}(\text { the.dishes })\left(e^{\prime}\right)\right) \\
=\lambda e . \exists p\left[\operatorname{meas}\left[\min \left(S_{\text {w.t.d. }}\right), \mathrm{p}\right] \geq \operatorname{meas}\left[\mathrm{p}, \max \left(S_{\text {w.t.d. }}\right)\right] \wedge\right. \\
\quad \operatorname{wash}(\text { the.dishes })(e) \text { w.r.t. } p]
\end{gathered}
$$

In (23b) the scale targeted by half is the scale of objects made available by the presence of the incremental theme the dishes. Since the dishes is a bounded, or quantized, incremental theme, the corresponding scale of objects is closed, a prerequisite for the successful application of half. Again, this analysis explains why VPs with unbounded or non-quantized incremental themes (e.g. dishes) cannot be modified by half - the scale of objects they induce are also unbounded, i.e. open, and thus are inappropriate targets for half. It should also be clear from (23) why half cannot target the external argument of the verb. Since the external argument is not a lexical argument of the verb and is introduced into the syntax and semantics above VP, the event description modified by half makes no reference to the external argument, leaving it unavailable for modification. I assume that adjectival passives such as (19b) are derived from an unaccusative syntax where the cake is the internal argument of bake (see section 3.1). Thus, the derivation of (19b) proceeds in exactly the same way as in (23), and existential closure of the event argument yields the event state described by the adjectival passive (taking states to be a type of event).

\subsection{Abstract Events}

The meaning for half in (22) relies on making reference to the point $\max \left(S_{F}\right)$ on the scale of objects, which is the maximal endpoint of the scale that is targeted by Filip's $\mathrm{MAX}_{\mathrm{E}}$ operator. However, making reference to the maximal endpoint should not presuppose the existence of a maximal event in the first place. Indeed, the sentence The girls half washed the dishes does not entail that all the dishes were eventually washed. This observation does not pose a real problem for this analysis, however. The point $\max \left(S_{F}\right)$ just makes reference to a maximal event, which remains abstract and possibly unrealized. This view is similar to an idea by 


\section{Promiscuous Modification and Scale Structure}

Moltmann (1997) that adverbs of completion (such as half, mostly, completely) actually denote relations between abstract and concrete events. Piñón (2005) in his analysis of adverbs of completion also makes reference to abstract maximal events embedded under a possibility operator. This idea perhaps suggests a possible intensional analysis for half and similar modifiers, but this idea will not be explored here.

\section{Concluding Remarks}

I have shown that the use of half as a modifier of verb phrases has a scalar meaning parallel with its use as an adjectival modifier as discussed by Kennedy and McNally (2005). This analysis explains the property of half that it appears to be able to modify certain constituents at a distance while in the syntactic position of modifying VPs that denote telic event descriptions. These long-distance effects are in fact an illusion since they ultimately derive from local modification at the VP level. VP event descriptions are associated with scales that are induced by the incremental theme argument. Since it is the theme argument that provides the scale targeted by half, this gives rise to the illusion of long-distance modification when in fact modification is strictly local at the VP level.

For now, the question of exactly how the scale of objects induced by the theme argument gets passed up to the VP level in the first place remains a mystery. Filip (2008) seems to hint that it has something to do with the object-event homomorphism, but provides no formal details. In Bochnak (2009) I suggest that part of the VP meaning is a degree argument that needs to be saturated in order to arrive at an event description. Thus, half supplies the degree argument with the value of the midpoint on a scale associated with the VP, i.e., the scale of objects. Likewise, Filip's $\mathrm{MAX}_{\mathrm{E}}$ operator saturates the degree argument by supplying it with the maximal value on the scale.

In addition, a scalar meaning for half provides a starting point for explaining the modifier's cross-categorial promiscuity. Since both gradable adjectives and event-denoting VPs are associated with scales, half is able to appear as a modifier of either of these categories, so long as the scales being targeted are fully closed. Further discussion of this matter in Bochnak (2009) extends the scalar analysis of half to its use in partitives and its evaluative use. In particular, it is argued that half also targets fully closed scales in these contexts as well, which accounts for the modifier's promiscuous behavior across various syntactic and semantic categories.

This scalar analysis of half as a VP modifier may be helpful to account for other puzzles that have surfaced in the literature. For example, as noted by Morzycki (2002) there is a series of other proportional modifiers that result in "ambiguities" due to perceived long-distance modification effects:

(24) a. The campus is completely nauseating.

b. Oklahoma is wholly Republican. 


\section{Ryan Bochnak}

In (24a) completely can be construed as either modifying the parts of the campus that are nauseating, or the degree to which the campus induces nausea, i.e., the scale of nauseating. Similarly in (24b) the use of wholly can either make a comment about the parts of Oklahoma that are Republican, or the extent to which the state is Republican. Treating these modifiers as parallel with half with respect to this behavior provides further evidence for their status as VP modifiers and not as floating quantifiers, notably because the modifiers in (24) are morphosyntactically marked as adverbs. While half has the same phonological shape no matter which category it modifies, these other modifiers are clearly marked as adverbs in (24), making it difficult to argue for an underlying representation where completely and wholly directly modify the campus and Oklahoma, respectively. Instead, if we analyze these modifiers as modifying the VP and having access to the scale of objects induced by the internal argument, we can easily account for the multiple readings of the sentences in (24) without having to posit underlying structures where DPs are syntactically modified by adverbs. Again, like the long-distance effects associated with half, those in (24) are actually derived from local modification of a VP that is associated with a scale of objects thanks to the verb's bounded incremental theme argument. (Although the categorical status of Republican as being derived from a verb is at best questionable, assuming that all adjectival passives are derived from an unaccusative syntax still yields the desired results for the perceived long-distance effects).

In addition, the counterparts of half in other languages have been shown to behave in similar ways with respect to multiple available readings. For example, Moltmann (1997) provides the following example from German:

Der Baum ist halb vertrocknet.

'The tree is half dried out.'

According to Moltmann this sentence has two readings: one in which the tree has reached the half-way point on the scale of dryness, and another in which only half of the tree is fully dried out. Moreover, as has been pointed out by Doetjes (1997) French beaucoup shows similar properties, displaying what she refers to as "quantification at distance:"

Jean a beaucoup lu de livres.
Jean AUX a.lot read of
'Jean read a lot of books.'

In (26), the most salient reading is one where beaucoup quantifies over the number of books read and does so at a distance, although there is also another reading where beaucoup quantifies over the number of book-reading events. Given the parallels between the available readings for sentences with half discussed in this paper and those for the sentences in (24)-(26), it seems likely that a unified analysis that treats all these modifiers as VP modifiers alongside half 


\section{Promiscuous Modification and Scale Structure}

would be able to account for the relevant facts, both in English and crosslinguistically. However, I leave this question to further study.

\section{Acknowledgments}

I would like to thank audiences at the Berkeley Linguistics Society meeting in February 2009 and at the University of Chicago LingLunch series at which this work was presented. I am especially grateful to Chris Kennedy and Peter Alrenga for their helpful comments and suggestions.

\section{References}

Bochnak, M. Ryan. 2009. Half as a promiscuous modifier. Ms., University of Chicago.

Doetjes, Jenny. 1997. Quantifiers and selection: On the distribution of quantifying expressions in French, Dutch and English. Ph.D. diss., Leiden University.

Dowty, David. 1991. Thematic proto-roles and argument selection. Language 67(3):547-619.

Filip, Hana. 2008. Events and maximalization. In S. Rothstein, ed., Theoretical and crosslinguistic approaches to the semantics of aspect, 217-256. Philadelphia: John Benjamins.

Geurts, Bart. 2006. Take 'five': The meaning and use of a number word. In S. Vogeleer and L. Tasmowski, eds., Non-definiteness and plurality, 311-329. Philadelphia: John Benjamins.

Ionin, Tania, and Ora Matushansky. 2006. The composition of complex cardinals. Journal of Semantics 23(4):315-360.

Jackendoff, Ray. 1996. The proper treatment of measuring out, telicity, and perhaps even quantification in English. Natural Language and Linguistic Theory 14(2):305-354.

Kennedy, Christopher, and Louise McNally. 2005. Scale structure, degree modification and the semantics of gradable predicates. Language 81(2):345-381.

Kratzer, Angelika. 2003. The event argument. Ms., University of Massachusetts, Amherst.

Krifka, Manfred. 1992. Thematic relations as links between nominal reference and temporal constitution. In I. Sag and A. Szabolsci, eds., Lexical matters, 29-53. Stanford, CA: CSLI.

Krifka, Manfred. 1998. The origins of telicity. In S. Rothstein, ed., Events and grammar, 197-235. Dordrecht: Kluwer.

Levinson, Stephen C. 2000. Presumptive meanings: The theory of generalized conversational implicature. Cambridge, MA: MIT Press.

Moltmann, Friederike. 1997. Parts and wholes in semantics. New York: Oxford University Press.

Morzycki, Marcin. 2002. Wholes and their covers. In B. Jackson, ed., Proceedings of Semantics and Linguistic Theory (SALT) XII, Ithaca, NY: CLC Publications. 


\section{Ryan Bochnak}

Piñón, Christopher. 2005. Adverbs of completion in an event semantics. In H. J. Verkuyl, H. de Swart, and A. van Hout, eds., Perspectives on aspect, 149-166. Dordrecht: Springer.

Sportiche, Dominique. 1988. A theory of floating quantifiers and its corollaries for constituent structure. Linguistic Inquiry 19(2):425-451.

Tenny, Carol. 1994. Aspectual roles and the syntax-semantics interface. Dordrecht: Kluwer.

M. Ryan Bochnak

University of Chicago

Department of Linguistics

1010 E. 59th St.

Chicago, IL 60637

bochnak@uchicago.edu 